\title{
A INFLUÊNCIA DA AUTOESTIMA NO DESEMPENHO ESCOLAR
}

\section{THE INFLUENCE OF SELF-ESTEEM ON SCHOOL PERFORMANCE}

\author{
Renata Furtado Horta ${ }^{1}$ \\ Marcelo Ferreira ${ }^{2}$
}

\begin{abstract}
RESUMO: O desempenho dos estudantes é afetado por aspectos cognitivos, relacionais e emocionais. No âmbito das emoções, a autoestima desempenha papel essencial na aprendizagem e no progresso social dos alunos. Para a construção de uma autoestima saudável, é estritamente necessário o envolvimento de familiares e equipe escolar, a fim de proporcionar um ambiente seguro e motivador para a criança e adolescente. Através de revisões bibliográficas, foi possível observar as definições de autoestima, dimensionar ações que impactam na autoestima dos alunos e conhecer teorias que apontam a importância da autoestima no ambiente escolar. Dentro do ensino de matemática, estudos identificaram os mais variados tipos de emoções relacionadas à realidade de cada aluno e percebe-se que, tanto as afetividades positivas quanto as negativas, influenciam no desempenho e nas relações escolares. A partir da discussão elaborada, é possível notar que a autoestima pode ser elevada a partir das ações da equipe escolar e da contextualização da vivência do aluno, sendo essencial o incentivo familiar e escolar para o desenvolvimento eficaz e significativo da aprendizagem.
\end{abstract}

PALAVRAS-CHAVE: Autoestima. Emoções. Ensino de Matemática.

\begin{abstract}
Student performance is affected by cognitive, relational and emotional aspects. In terms of emotions, self-esteem plays an essential role in students' learning and social progress. To build a healthy selfesteem, the involvement of family members and school staff is strictly necessary, in order to provide a safe and motivating environment for children and adolescents. Through literature reviews, it was possible to observe the definitions of self-esteem, dimension actions that impact on the students' self-esteem and learn about theories that point to the importance of self-esteem in the school environment. Within the teaching of mathematics, studies have identified the most varied types of emotions related to the reality of each student and it is clear that both positive and negative affectivities influence performance and school relationships. From the elaborated discussion, it is possible to notice that self-esteem can be increased from the actions of the school team and the contextualization of the student's experience, with family and school encouragement being essential for the effective and meaningful development of learning.
\end{abstract}

KEYWORDS: Self-esteem. Emotions. Mathematics teaching.

O desempenho dos estudantes é afetado por aspectos cognitivos, relacionais e, principalmente, pelos aspectos emocionais. No campo das emoções, a autoestima desempenha um papel crucial, o que é reforçado pela vasta literatura produzida na área. Embora o sucesso acadêmico seja o objetivo monitorado de forma regular e tratado como

\footnotetext{
1 Universidade Federal do Triângulo Mineiro. E-mail: re.horta@yahoo.com.br https://orcid.org/0000-0002-8832-3415

2 Universidade Federal do Triângulo Mineiro. E-mail: marcelo.ferreira@uftm.edu.br

(iD) https://orcid.org/0000-0002-6854-1837

- Informações completas da obra no final do artigo
} 
um forte indicador de qualidade de vida escolar (SLAVIN, 2006), o bem-estar psicológico é considerado decisivo para o sucesso dos alunos. Este bem-estar depende fortemente da autoestima, considerada elemento chave na educação (FERKANY, 2008; HUMPHREY, 2004).

A aprendizagem, segundo alguns estudiosos, é muito mais que apenas comportamentos nela estão implícitos conceitos psicológicos importantes (MENDES et al., 2017). Dentre os conceitos psicológicos, a autoestima tem sido vista como um dos indicadores sociais de importância fundamental para a compreensão do crescimento e do progresso social, tornando-se ponto essencial a ser observado em um desenvolvimento pessoal sadio e completo em todas as áreas da vida. Além disso, entende-se que há uma conexão estritamente importante entre autoestima e rendimento escolar (DIOGO, 2009).

Nesse contexto, tem-se uma efetiva preocupação quanto à importância da autoestima e a influência que o professor e a equipe escolar possuem na vida e no desempenho de cada aluno. É fato que faz parte do papel da equipe escolar, possibilitar que o aluno aprenda em um ambiente em que se sinta seguro e motivado. As escolas possuem uma atmosfera desafiadora para a aprendizagem dos alunos e, para que o aluno aprenda de forma eficaz, se faz necessário o desenvolvimento de um ambiente atraente, positivo, entusiasta e encorajador para estimular gestores, professores e alunos (KOPFHAMMER, 1992).

Atualmente, através de estudos detalhados, identificaram os mais variados tipos de emoções relacionadas à realidade de cada aluno em ambientes escolares e percebe-se que, tanto as afetividades positivas quanto as negativas, influenciam no aprendizado. Sendo assim, este estudo tem como objetivo propor aos professores e a equipe escolar, alguns métodos que possibilitem o aumento da autoestima nos alunos e, como consequência, haja o desenvolvimento da aprendizagem em todo âmbito escolar, com foco, neste estudo, na aprendizagem de matemática.

No que se refere ao ensino de matemática no Brasil, nota-se que a abordagem das emoções e a afetividade não são triviais dentro das salas de aula e dessa forma, o raciocínio matemático se mostra deficiente na população brasileira. Segundo estudos, a autoestima possui influência no desempenho matemático e atua de forma a caracterizar a experiência de aprendizagem do aluno. Emoções como prazer, vergonha, orgulho e ansiedade estão estritamente relacionados ao desempenho matemático dos alunos (LIMA, 2012). 
Dados da literatura demonstram que existe uma relação direta entre autoestima e a aprendizagem. O aluno que possui uma autoestima positiva aprende mais feliz e, com maior felicidade, atinge níveis mais altos em sua vida pessoal e profissional. Já o aluno que tem a autoestima baixa, tende a ser relapso em sua vida escolar e profissional e, este aluno que tem a autoimagem distorcida, tende a demonstrar desinteresse na aprendizagem e mau comportamento escolar (BEAN et al., 1995).

Mediante as revisões bibliográficas, foi possível observar análises descritivas multidimensionais dos quais se obtiveram a interação de grupos de pessoas ligados às mesmas afetividades, e com isso, trouxeram uma concepção destinada à determinada direção, permitindo afirmar que a autoestima pode ser determinante no próprio desempenho. Desse modo, este estudo indica que alguns métodos podem melhorar 0 desempenho matemático, possibilitando autonomia no processo de aprendizagem e nas escolhas pessoais e profissionais.

Acredita-se que a autoestima se inicia na infância, sofrendo influências dos familiares, pais/responsáveis, professores e colegas. Quanto mais nova a criança, mais influências das pessoas próximas a ela definem sua autoestima. Já na adolescência, os hormônios e os colegas tendem a influenciar muito mais do que as pessoas mais próximas. Uma das mais importantes e desafiadoras funções da escola nos últimos tempos é tornar o ambiente escolar um ambiente positivo e encorajador, principalmente para o adolescente. Muitos alunos entram na escola com sua imagem já distorcida, sendo muitas vezes em seu ambiente familiar e doméstico expostos a violências verbais, físicas, sexuais, intelectuais, alcoolismo, prostituição, drogas, entre outras situações de abandonos e desafeto que muito prejudica e agride o desenvolvimento da criança e do adolescente. As raízes multifacetadas do desejo de se melhorar no ambiente escolar e na vida social de um adolescente, estão intimamente ligadas ao desempenho de sua vida pessoal. A autoestima pode ser mudada e aprendida, portanto, pode ser ensinada, tornando-se um pré-requisito necessário para o desempenho escolar (KOPHAMMER, 1992).

Este estudo é direcionado ao público do Ensino Fundamental e Médio, a fim de proporcionar aos alunos autonomia no processo de aprendizagem e melhora no seu desempenho acadêmico. Inserindo alguns métodos para a melhora da autoestima, o aluno se torna parte fundamental no processo de ensino e aprendizagem, e se porta, não apenas 
como objeto a ser estudado, mas sujeito participante de um processo dinâmico em que as possibilidades são construídas num movimento individual e coletivo, no qual o docente desenvolve papel fundamental.

A autoestima é definida como o conhecimento do indivíduo sobre seu próprio valor, sendo então uma apreciação de seus princípios e importância, e a responsabilidade por si mesmo e para com os outros. Uma autoestima saudável inclui uma vida com mais amor, trabalho, lazer. Portanto, é um componente avaliador do autoconceito (BRANDEN, 1997). Diante de uma autoestima alta, a pessoa demonstra as seguintes características:

1- Age de forma independente;

2- É mais responsável;

3- Tem orgulho do que faz;

4- Não tem medo de mostrar seus sentimentos;

5- Aprende a viver com frustrações;

6- Consegue influenciar outras pessoas com seu entusiasmo.

Já a pessoa que possui uma autoestima baixa tende a:

1- Não aceitar suas qualidades;

2- Não se sente valorizada;

3- Tem medo de expor seus sentimentos;

4- É muito influenciada por outras pessoas;

5- Não sabe viver com frustrações;

6- Tende a culpar outras pessoas por suas fraquezas ou erro se torna defensiva diante dos problemas.

Alguns fatores ajudam a determinar e desenvolver a autoestima de uma pessoa, dentre eles, o status socioeconômico, altura, peso, média de notas na escola, religião e participação na sociedade. Entre os adolescentes, estes fatores são fundamentais no desenvolvimento de uma autoestima positiva. A autoestima tem em seu significado a relação entre a imagem ou a opinião que um indivíduo faz de si mesmo, e se constrói a partir de suas experiências, sendo a chave para o sucesso ou fracasso. Além disso, caracteriza-se pela soma do autorrespeito com a autoconfiança e reflete com a capacidade em lidar com os desafios da vida e o direito de ser feliz. Esse processo deve incluir lidar com frustrações, negações, informações que geram desonestidades, irresponsabilidades, 
incompreensão, sentimentos e emoções que provocam no indivíduo conflitos internos que afetam diretamente sua autoestima.

No livro Seis Pilares da Autoestima (BRANDEN, 1997), há a demonstração de metodologias práticas de como se pode desenvolver com os alunos uma autoestima alta, tendo como práticas: o viver conscientemente, a autoaceitação, a autorresponsabilidade, a autoafirmação, a vida com propósito e a integridade pessoal. Além disso, os ensinamentos dos professores e da equipe escolar podem refletir em toda a vida do aluno e nos dá algumas ideias de como esta autoestima pode ser aprendida e como melhorar 0 desempenho escolar (KOPFHAMMER, 1992), ver Figura 1.

Figura 1. Dez etapas para ajudar os alunos a aumentarem sua autoestima de acordo com Kopfhammer (1992).

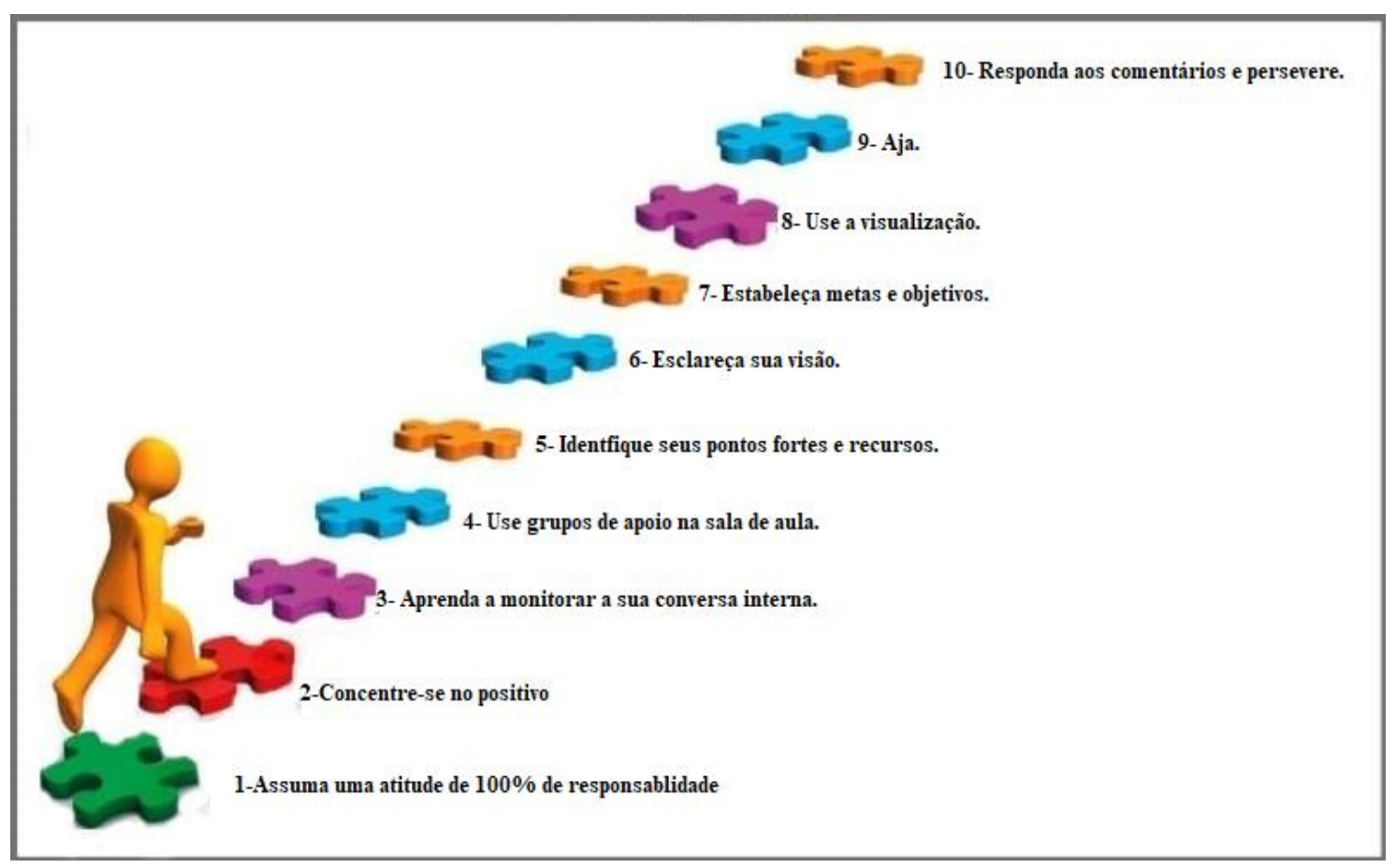

Fonte: Os autores, adaptado de Kopfhammer (1992).

Como dito anteriormente, acredita-se que a autoestima se inicia na infância, porém pode ser alterada independentemente da idade. Logo, cada professor precisa estar disposto a ir atrás dos métodos, passos e etapas que leve o aluno a estar disposto e interessado em aprender e sair de sua zona de conforto (KOPFHAMMER, 1992). Não é um trabalho fácil, mas é necessário para mudar a educação como um todo. Quanto maior a autoestima, maior o desempenho acadêmico e o sentimento de motivação para aprender, tendo um sentimento de prazer e orgulho na matemática. Por outro lado, o aluno com 
autoestima baixa tem vergonha e ansiedade na matemática, visto que seu desempenho é inferior ao esperado (LIMA, 2012).

A aprendizagem matemática é vista com uma especificidade (ARAUJO et al., 2003), e diante desta realidade, foram feitas pesquisas que mostram que a afetividade e a relação de confiança com os professores refletem na efetiva aprendizagem dos alunos. Segundo estudos, a autoestima é dinâmica e requer um longo processo para construí-la. Para isto, o autor usou em sua pesquisa dois grupos experimentais em escolas de classe alta, média e baixa, sendo um de controle em que recebiam todo o suporte do professor com uma abordagem aberta e o outro grupo apenas uma abordagem tradicional e, como conclusão, obteve um resultado em que o grupo que recebia uma abordagem aberta teve um desempenho muito melhor do que aqueles que eram ensinados de uma forma convencional. Os alunos aumentaram a criatividade matemática e a capacidade de pensar, melhorando significativamente sua autoestima (FATAH et al., 2016).

Outros estudos apresentam conexões entre os aspectos cognitivos e afetivos na aprendizagem e, em especial, na aprendizagem dos conceitos de matemática no $1^{\circ}$ ciclo, do $1^{\circ}$ ao $5^{\circ}$ ano do Ensino Fundamental. Na perspectiva dos estudos na linha construtivista, a aprendizagem efetiva é uma atividade ativa de resolução de problemas e ao se resolver um problema, as emoções que mais se observa nos alunos são satisfação ao encontrar a solução ou frustração quando não a encontram. Os dados desta pesquisa mostram que a relação de confiança com os professores reflete de forma essencial na aprendizagem dos conhecimentos matemáticos (HAZIN et al., 2010). O professor que ensina de forma ativa e atrelada a um ambiente familiar estimulante, influencia na aprendizagem da matemática. Estabelecer relações entre o conteúdo e a realidade dos alunos, inserindo-o como parte fundamental no processo, não como objeto a ser estudado, mas como sujeito participante de um processo dinâmico, onde as possibilidades são construídas num movimento individual e coletivo é fundamental para a construção da autoestima e, consequentemente, da aprendizagem. A participação dos alunos favorece a aprendizagem, estimula a disciplina e potencializa o interesse pelo saber matemático, propondo uma autoestima positiva que propõe uma participação mais ativa dos alunos (NEVES; CARVALHO, 2006).

Teorias demonstram a importância da autoestima e como ela surge na escola. Conforme podemos ver na Figura 2, a teoria da interação simbólica. 
Figura 2. Teorias sobre o desenvolvimento de autoestima na escola.

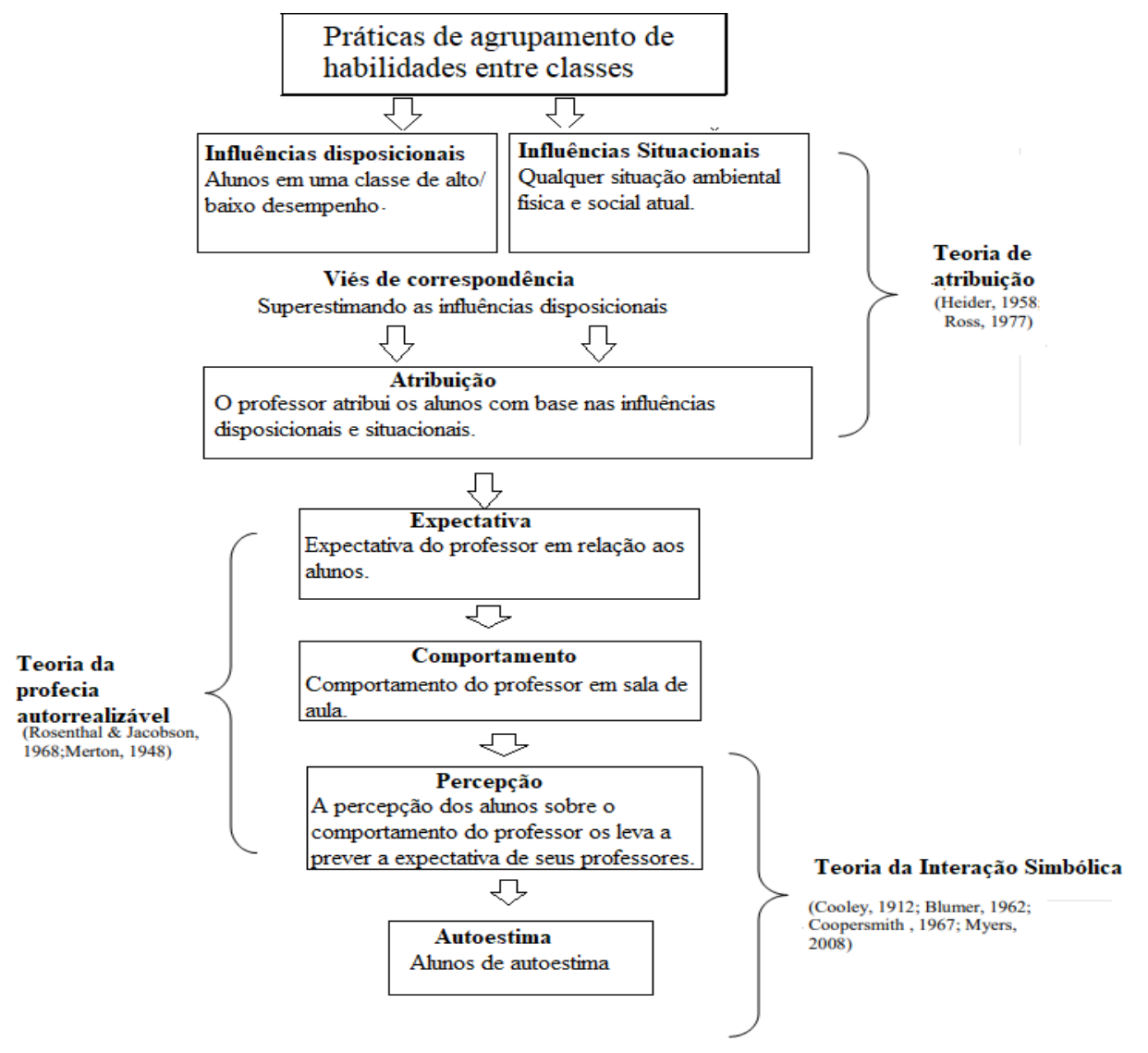

Fonte: Prihadi e Chua (2012), tradução nossa.

Esta teoria aponta que a expectativa dos professores é percebida pelos estudantes e possui influência indireta na sua autoavaliação, ou seja, quando o aluno percebe que seu professor tem expectativas positivas quanto ao seu desempenho, ele tem uma maior interesse e se dedica mais aquele estudo, já quando o aluno percebe que seu professor não tem expectativas positivas quanto ao seu desempenho, ele se sente desmotivado a aprender aquele conteúdo, já que indiretamente o professor insinuou que ele não conseguiria se destacar neste estudo (PRIHADI; CHUA, 2012).

A segunda teoria apresentada é a teoria da profecia autorrealizável, que traz como exemplo um professor que leciona para dois ou mais estudantes da mesma família, irmãos ou primos e acaba, de forma inconsciente, promovendo uma tensão entre o estudante e os demais alunos da sala. O professor por ter dado aula para um estudante da família que era brilhante na escola, tende a esperar o mesmo dos outros mais novos, e com isso, possui um comportamento para com aquele aluno mais novo, de atenção, reforço e cuidado, 


\section{ENSIN@UFMS 2021}

ISSN 2525-7056

possibilitando que o aluno mais novo se sinta motivado e com entusiasmo. Dessa forma, se realiza a "profecia" do professor que aquele aluno seria um estudante brilhante.

A terceira e última teoria, é a teoria da atribuição, conforme mostra a Figura 3.

Figura 3. Exemplo Teoria da Atribuição.

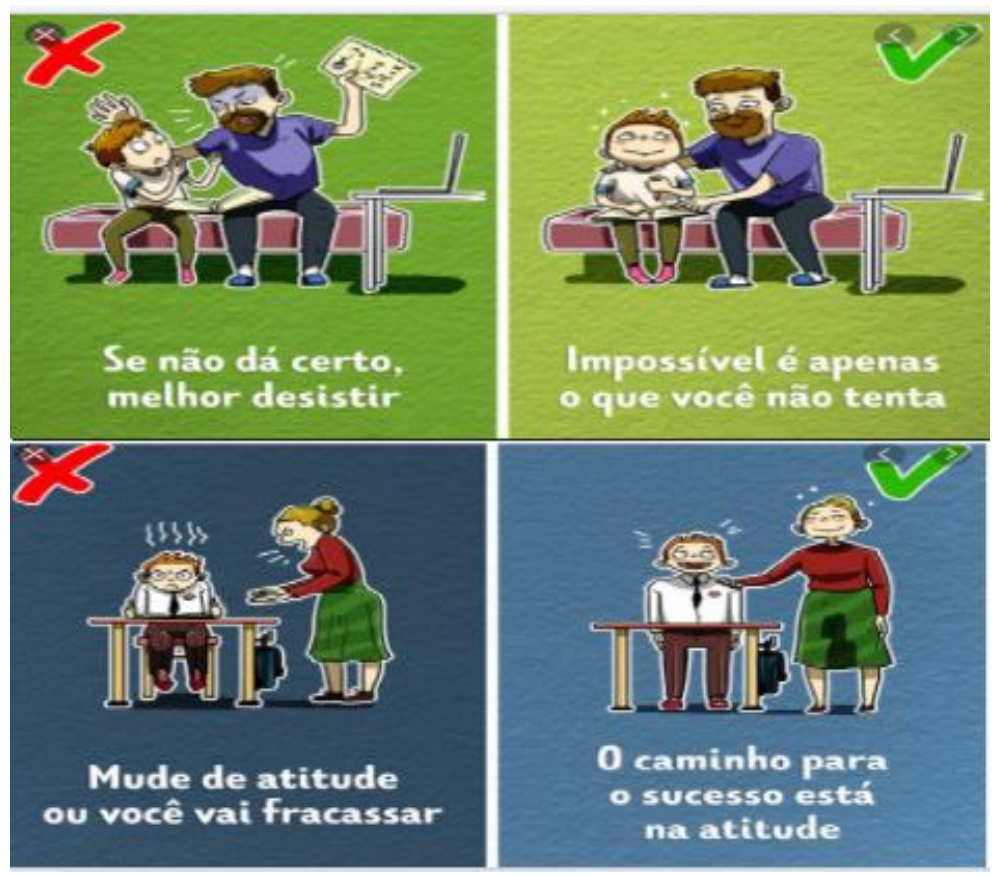

Fonte:https://incrivel.club/inspiracion-psicologia/10-frases-para-reforzar-la-autoestima-de-tuhijo-al-hacer-las-tareas-escolares-900210/ (adaptada).

Teoria em que um fato disposicional ou situacional indica a reação desfavorável ou simpática, como por exemplo, um estudante que é rude com seus colegas recebe a atribuição situacional desfavorável de ser uma pessoa sem educação e odiado pelo restante dos colegas; caso a atribuição fosse simpática, o aluno poderia aprender a ser mais gentil (PRIHADI; CHUA, 2012). Isto acontece também quando se formam grupos selecionados pela aptidão no conteúdo e há a rotulação dos alunos.

Conforme é apresentado, a autoestima se mostra como parte fundamental no desempenho escolar. Através de metodologias ativas que proporcionem a participação dos alunos, é possível desenvolver a confiança e o interesse em uma aprendizagem mais significativa. A autoestima se dispõe como preditora do desempenho escolar, em que o aluno que confia em seus valores e capacidades, se permite envolver no processo e na concretização da aprendizagem. A busca pela construção de uma autoestima saudável em crianças e adolescentes possibilita ampliar não só o interesse dos alunos nos conteúdos 


\section{ENSIN@UFMS 2021}

ISSN 2525-7056

escolares, mas também contribui para realizações futuras em sua vida pessoal e profissional. Cabe salientar que o papel do professor está em ajudar os alunos a enxergar suas potencialidades e permitir que sua participação seja efetiva na aprendizagem e no desempenho escolar.

\section{Referências}

ARAÚJO, C. R. et al. Affective Aspects on Mathematics Conceptualization: From Dichotomies to an Integrated Approach. International Group for the Psychology of Mathematics Education, v. 2, p. 269-276, 2003.

BEAN, R. et al. Adolescentes Seguros: Como aumentar a autoestima dos jovens. São Paulo: Gente, 1995.

BRANDEN, N. Auto-estima e os seus seis pilares. Tradução Vera Caputo. São Paulo: 3 ed., 1997.

DIOGO, F. V. Relação familiar e autoestima. Investigação, São Paulo, v. 9, n. 1, p. 1724, jan./abr. 2009.

FATAH, A. et al. Open-Ended Approach: An Effort in Cultivating Students' Mathematical Creative Thinking Ability and Self-Esteem in

Mathematics. JournalonMathematicsEducation, v. 7, n. 1, p. 11-20, 2016.

FERKANY, M. The educationalimportanceof self-esteem.

JournalofPhilosophyofEducation, 2008.

HAZIN, I; FRADE, C; FALCÃO, J. T. R. Autoestima e desempenho escolar em matemática: contribuições teóricas sobre a problematização das relações entre cognição e afetividade. Educar, n. 36, p. 39-54, 2010.

HUMPHREY, N.The Death oftheFeel-Good Factor: Self-Esteem in theEducationalContext. SchoolPsychologylnternational, 2004.

KOPFHAMMER, P. H. Research and methods of improving: elementary school students' self-esteem. MarquetteUniversity, 1992. Disponível em:

https://www.marquette.edu/library/theses/already_uploaded_to_IR/kopfh_p_1992.pdf.

Acesso em: 15 ago 2021.

LIMA, M. C. F. Emoções de desempenho na matemática e suas relações com autoconceito acadêmico, autoimagem e autoconsciência. Dissertação - Universidade Federal de Pernambuco - Programa de pós-graduação em psicologia. Recife, 2012. 
MENDES, D. C; CASTELANO, K. L; MARTINS, L. M; ANDRADE, Claudia Caixeta Franco. A influência da autoestima no desempenho escolar. Revista Educação em Debate, Fortaleza, ano 39, n. 73, p. 9-21, jan./jun. 2017.

NEVES, M. C; CARVALHO, C. A importância da afectividade na aprendizagem da

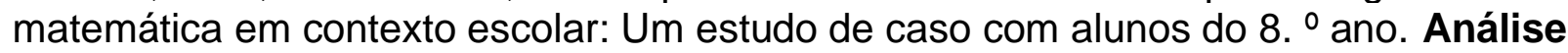
Psicológica, v. 24, n. 2, p. 201-215, 2006.

PRIHADI, K; CHUA, M. Students' Self-Esteem at School: The Risk, the Challenge, and the Cure. JournalofEducationand Learning. Vol. 6, p. 1-14, 2012.

SLAVIN, R. E. AbilityGroupingandStudentAchievement in ElementarySchools. Review ofEducationalResearch, 2006.

\title{
NOTAS
}

\section{IDENTIFICAÇÃO DO TEXTO}

O presente texto é um recorte da dissertação de Mestrado Profissional em Matemática em Rede Nacional (Profmat) em desenvolvimento na Universidade Federal do Triângulo Mineiro (UFTM), elaborada sob orientação do Professor Dr. Marcelo Ferreira.

\section{IDENTIFICAÇÃO DE AUTORIA}

Renata Furtado Horta. Discente do Programa de Mestrado Profissional em Matemática em Rede Nacional (PROFMAT). Universidade Federal do Triângulo Mineiro (UFTM), Uberaba, Minas Gerais, Brasil.

E-mail: re.horta@yahoo.com.br.

(D) https://orcid.org/0000-0002-8832-3415

\begin{abstract}
Marcelo Ferreira. Doutor em Matemática Aplicada pela Universidade Estadual Paulista (UNESP), São José do Rio Preto, SP. Orientador do Programa de Mestrado Profissional em Matemática em Rede Nacional (PROFMAT). Universidade Federal do Triângulo Mineiro (UFTM), Uberaba, Minas Gerais, Brasil.

E-mail: marcelo.ferreira@uftm.edu.br

(iD) https://orcid.org/0000-0002-6854-1837
\end{abstract}

\section{AGRADECIMENTOS}

Agradecemos aos idealizadores do Profmat, e ao corpo docente da Universidade Federal do Triângulo Mineiro (UFTM).

\section{FINANCIAMENTO}

O presente trabalho foi realizado com apoio da Coordenação de Aperfeiçoamento de Pessoal de Nível Superior - Brasil (CAPES) - Código de Financiamento 001.

\section{CONSENTIMENTO DE USO DE IMAGEM}

Não se aplica.

\section{APROVAÇÃO DE COMITÊ DE ÉTICA EM PESQUISA \\ Não se aplica.}

\section{LICENÇA DE USO}

Autores mantêm os direitos autorais e concedem à revista ENSIN@ UFMS - ISSN 2525-7056 o direito de primeira publicação, com o trabalho simultaneamente licenciado sob a Licença Creative Commons Attribution (CC BY-NC-SA 4.0), que permite compartilhar e adaptar o trabalho, para fins não comerciais, reconhecendo a autoria do texto e publicação inicial neste periódico, desde que adotem a mesma licença, compartilhar igual. 


\section{EDITORES}

Patricia Helena Mirandola Garcia, Eugenia Brunilda Opazo Uribe, Gerson dos Santos Farias.

\section{HISTÓRICO}

Recebido em: 14/09/2021 - Aprovado em: 12/12/2021 - Publicado em: 15/12/2021.

\section{COMO CITAR}

HORTA, R. F; FERREIRA, M. A Influência da Autoestima no Desempenho Escolar. Revista ENSIN@ UFMS, Três Lagoas, v. 2, número especial, p. 276-286. 2021. 